\title{
A Systematic Review of Complications and Failures Associated with Medial Patellofemoral Ligament Reconstruction for Recurrent Patellar Dislocation
}

\author{
Jay N. Shah, MD, Jennifer S. Howard, PhD, ATCy, David C. Flanigan, MDz, Robert H. \\ Brophy, MD§, James L. Carey, MD, MPH", and Christian Lattermann, MD ${ }^{*},\{$ \\ "Department of Orthopaedic Surgery and Sports Medicine, University of Kentucky, Lexington, \\ Kentucky \\ yepartment of Rehabilitation Sciences, University of Kentucky, Lexington, Kentucky \\ ${ }^{z}$ Cartilage Restoration Program, Sports Health and Performance Institute, Department of \\ Orthopedics, The Ohio State University, Columbus, Ohio \\ $\S$ Department of Orthopedic Surgery, Washington University School of Medicine, St Louis, \\ Missouri \\ "Penn Center for Advanced Cartilage Repair and Osteochondritis Dissecans Treatment, \\ Perelman School of Medicine, University of Pennsylvania, Philadelphia, Pennsylvania
}

\begin{abstract}
Background-Patellofemoral instability affects activities of daily living and hinders athletic participation. Over the past 2 decades more attention has been paid to medial patellofemoral ligament reconstruction (MPFL) for treatment of recurrent patellar dislocations/subluxations. Numerous techniques have been reported; however, there is no consensus regarding optimal reconstruction.
\end{abstract}

Purpose-This study sought to report on the various techniques for MPFL reconstruction described in the literature and to assess the rate of complications associated with the procedure.

Study Design-Meta-analysis.

Methods-A systematic review of the literature was performed in early October 2010 using keywords "medial patellofemoral ligament," "MPFL", "reconstruction," "complication(s)," and "failure(s)". Articles meeting the inclusion criteria were reviewed. Graft choice, surgical technique, outcome measures, and complications were recorded and organized in a database. Descriptive statistical analysis was performed on the data collected.

Results-Twenty-five articles were identified and reviewed. A total of 164 complications occurred in 629 knees (26.1\%). These adverse events ranged from minor to major including patella fracture, failures, clinical instability on post-operative examination, loss of knee flexion, wound complications, and pain. Twenty-six patients returned to the operating room for additional procedures.

\footnotetext{
'Address correspondence to Christian Lattermann, MD, University of Kentucky, Department of Orthopaedic Surgery and Sports Medicine, 740 South Limestone, K435, Lexington, KY 40536 (clattermann@ gmail.com)..

Conflicts of Interest—none

The authors declared that they have no conflicts of interest in the authorship and publication of this contribution.
} 
Conclusions-Medial patellofemoral ligament reconstruction has a high rate of success for patients with patellofemoral instability; however, the complication rate of $26.1 \%$ associated with this procedure is not trivial. This study quantified complications and documented the variety of complications reported in outcomes-based literature.

\section{Keywords}

patellofemoral instability; medial patellofemoral ligament; MPFL reconstruction complications; MPFL reconstruction techniques; patellar dislocation

\section{Introduction}

Recurrent patellar instability is a disabling condition that tends to affect younger individuals ${ }^{17,21}$. While primary dislocations frequently happen due to a direct high energy trauma, low energy patella dislocations often occur in individuals with preexisting variants such as increased quadriceps angle, generalized ligamentous laxity, family history, ${ }^{4}$ trochlear dysplasia, or patella alta. ${ }^{5}$

The stability of the patella during motion is controlled by soft tissue and bone anatomy. The bony architecture of the patellofemoral joint predominantly guides the patella during higher flexion angles, while the soft tissue restraints stabilize the patella near extension. ${ }^{22}$ The primary soft tissue restraint for lateral subluxation of the patella near extension is the medial patellofemoral ligament (MPFL). The MPFL resides in layer 2 of the medial aspect of the knee, deep and slightly distal to the vastus medialis. It originates superoposterior to the medial femoral epicondyle, about $1 \mathrm{~cm}$ distal to the adductor tubercle, ${ }^{29}$ in the depression formed between the adductor tubercle and the medial epicondyle. ${ }^{3}$ The MPFL may form an arch with the superficial medial collateral ligament ${ }^{31}$ and inserts laterally over the proximal two-thirds of the medial patella in a fan-like fashion. ${ }^{29,31,42}$ The ligament tightens in full extension and loses tension upon flexion. Patellar stabilization within the normal trochlea occurs at 15 to 20 degrees flexion. ${ }^{2}$ Primary patella dislocations often lead to a disruption of the MPFL.

Even though the treatment of patellar instability has evolved significantly over the past 2 decades, consistent surgical technique to treat recurrent patella dislocations remains unclear. Nevertheless, the reconstruction of the MPFL has become a popular procedure for the treatment of these patients. ${ }^{7,11,20}$ The literature contains numerous methods of MPFL reconstruction for treatment of patellar dislocation with variations in graft choice, patellar fixation, femoral fixation, graft tension, and angle of knee flexion at time of fixation. The literature also recommends various fixation methods as well as different anatomic and nonanatomic reconstruction techniques. One of the most important aspects of evaluating a new procedure is assessing the complications and problems that are unique to each technique. Our interest, therefore, was to query the current literature with regards to the rate and type of post-operative complications after MPFL reconstruction. A secondary objective was to potentially identify specific techniques or practices that may have a higher complication rate than others. In order to accomplish this goal we performed a critical review of the literature.

\section{Methods}

Two independent authors performed a systematic review of the MPFL reconstruction literature. The search was performed in the first week of October 2010. The following databases were utilized: Medline, PubMed, CINAHL, SPORTDiscus, and Cochrane Collaboration of Systematic Reviews. Search terms utilized included "medial patellofemoral ligament," "MPFL," "reconstruction," complication(s)," and "failure(s)." This included all 
studies with Level I to IV evidence (Oxford Centre for Evidence Based Medicine adapted by the American version of the Journal of Bone and Joint Surgery $)^{30}$. To be included articles were required to report some form of data relating to complications or graft failures. Articles with questionable relevance to this study were discussed with the senior author. In addition, the first author evaluated the reference sections of selected studies for potentially inclusive articles missed during the initial search. Included articles were reviewed twice making note of graft choice, surgical technique (attention paid to patellar fixation, femoral fixation and location of graft, and adjuvant procedures), outcome measures, and complications. The authors of this study defined complications as any negative outcome including persistence of preoperative symptoms, returning to the operating room, symptomatic hardware, loss of arc range of motion, pain, and so on. The authors considered all complications mutually exclusive unless otherwise stated. These findings were recorded and organized in a database.

The pooled complication rate was calculated by pooling the number of reconstructed knees and reported complications across all included studies. Additionally, for each study the overall complication rate was calculated and then separate complication rates were calculated for recurrent subluxation/dislocation and post-operative reports of apprehension, patellar hypermobility, or episodic unstable feelings at final follow-up without frank subluxation or dislocation of the patella. To examine the variability in the reporting complications, the mean complication rate reported across studies was calculated.

Pooled complication rates were also used to descriptively examine the effect of surgical technique on complications. Complication rates were calculated to compare studies using sutures and/or suture anchors to those utilizing patellar tunnels for graft fixation. For the purposes of this review, any technique that required the creation of one or more patellar tunnels was classified as a "tunnel" technique and all other techniques were classified as "suture" techniques. Additionally, cumulative complication rates were compared for studies performing graft fixation at less than $60^{\circ}$ of knee flexion and those performing fixation at $60^{\circ}$ or greater knee flexion. Because of a lack of randomized controlled trials and significant heterogeneity between studies, neither risk ratios nor odds ratios could be calculated to directly compare the risk of complications between surgical techniques.

\section{Results}

\section{Article Selection}

The initial search yielded 146 articles. Forty three articles were eliminated because they did not address MPFL reconstruction (described other procedures). Anatomy studies (cadaveric, imaging, computational, histology) comprised 27 papers. Fifteen studies described surgical technique without reporting outcomes for any patients. Six papers were deemed case reports without information regarding clinical outcomes within a cohort. Review articles eliminated 9 reports. Seven papers did not have an abstract and were categorized as editorials or letters. Non-English papers eliminated 10 studies. Three articles reported on MPFL repair instead of reconstruction, and 1 article was excluded for failure to comment or report on complications or graft failures. After elimination of the above articles, we reviewed 25 scientific articles describing MPFL reconstruction with clinical outcomes.1, 6, 8-10, 12-16, 18, 19, 23-28, 32, 33, 36, 38, 40, 41, 43 See Figure 1 for a flowchart.

\section{Study Descriptives}

Table 1 outlines the study design, patient population, and complications associated with each article. No articles explicitly declared a conflict of interest. An expanded version of this table, detailing techniques involved in each study, is available as an online Appendix to this article (http://ajs.sagepub.com/supplemental/). Based on the Oxford Centre for Evidence 
Based Medicine criteria, no studies were classified as Level I evidence, 2 studies were Level II, 6 studies were Level III, and 17 studies were Level IV. A total of 629 knees underwent MPFL reconstruction. Men accounted for $242(40.5 \%)$ of the 597 patients. The average age for all patients was approximately 24 years with a range of 6 to 55 years ( 23 of 25 studies reported average age, others provided a median age). The average follow-up was approximately 47 months with a range of 3 to 204 months ( 23 of 25 studies reported average follow-up, others indicated median follow up or a minimum of 2 years' follow-up). One article noted patients with less than 11 months of follow-up ${ }^{23}$ but did not specify how many. Most studies did not report specific findings for preoperative instability. Diagnosis of patellar instability was based on history (e.g., multiple patellar dislocations, anterior knee pain), physical (e.g., patellar apprehension, patellar hypermobility), and radiographic findings (e.g., stress shift ratios, patella alta). Additional procedures augmenting the MPFL reconstruction included lateral retinacular release (LRR), tibial tubercle transfer (TTT), retinacular plication, vastus medialis obliquus (VMO) advancement, and chondroplasty. Two hundred thirty knees had LRR, 76 knees had TTT, and 67 knees had medial retinacular plication or VMO advancement. Four articles reported performing chondroplasty on a total of 22 patients; however, multiple articles reported various articular surface procedures without providing specific numbers.

Patellar fixation techniques varied amongst the studies. Seventeen studies used patellar tunnels ranging in size from $2.4 \mathrm{~mm}$ to $4.5 \mathrm{~mm}$ in diameter. Four studies used a single transverse tunnel drilled to 2 different diameters (larger diameter medially). Five articles had the patellar tunnel exiting anteriorly instead of laterally. Four articles investigated using dual tunnels separated by a bone bridge; one of these used dual tunnels that exited at the anterior patella. Sevens papers report using suture or suture anchors without patellar tunnels for lateral fixation of the MPFL graft. One article maintained the quadriceps tendon insertion on the superior pole of the patella for its lateral fixation.

Complication rates for individual studies varied greatly, ranging from $0 \% 24$ to $85.2 \%{ }^{26}$ with a mean complication rate of $25.7 \pm 21.3 \%$ across all included studies. The mean rates of recurrent subluxation/dislocation $(4.3 \pm 5.6 \%)$ and continued hypermobility/apprehension without subluxation or dislocation $(7.7 \pm 12.3)$ were also highly variable.

\section{Occurrence of Complications}

A total of 164 complications occurred in 629 knees (26.1\%). These adverse events ranged from minor to major. Broad classifications of failures included patella fracture, returning to the operating room, findings of clinical instability on post-operative exam, loss of knee flexion, wound complications, and pain. Four patients in 3 different studies sustained patella fracture after the initial procedure. Each of the studies used transpatellar tunnels where the graft was passed from the medial patellar aperture through the patella for lateral fixation. One author used a 3.2-mm transverse patellar tunnel, another used a $4.5-\mathrm{mm}$ patellar tunnel that exited anteriorly, and the last study used two $4.5 \mathrm{~mm}$ transverse tunnels separated by a 10- to 15 -mm bone bridge.

A total of 26 patients returned to the operating room for additional procedures. These procedures included addressing residual subluxation or dislocation $(n=6)$, excising a patellar fragment after fracture $(\mathrm{n}=1)$, manipulations under anesthesia to correct flexion loss $(n=9)$, removal of symptomatic hardware $(n=7)$, evacuation of hematoma $(n=1)$, and irrigation/debridement of surgical site infection $(n=2)$. Symptomatic hardware consistently occurred at the point of medial fixation on the femur.

There were 23 (3.7\%) knees that were deemed clinical failures at final follow up across 11 different studies. These patients experienced additional subluxation/dislocation after 
reconstruction of the MPFL. Five techniques passed the graft through a patellar tunnel that started medially and exited anteriorly or laterally. The graft was either folded the graft over the anterior patella or passed through a second patellar tunnel of similar orientation. The authors of this review generically classified this technique as a "looped" graft. Three articles used sutures or anchors without bone tunnels for patellar fixation of the graft. This was classified as suture fixation. One article used a transverse patellar tunnel and sutured the graft into the lateral retinaculum. One article used quadriceps tendon autograft for MPFL reconstruction while maintaining its insertion on the superior pole of the patella for its lateral "fixation." 12 The final article did not indicate which group sustained recurrent instability. ${ }^{9}$ Post-operative exams showed 52 of 629 knees (8.3\%) that still experienced apprehension, patellar hypermobility, or episodic unstable feelings at final follow-up without frank subluxation or dislocation of the patella. Because of the heterogeneity of defining failure amongst all the reports, the authors of this study classified patellar hypermobility/apprehension as a separate entity from redislocation/resubluxation.

Twenty-two knees had residual flexion loss at final follow-up, nine of which underwent manipulation under anesthesia. Wound complications occurred in 13 knees. These included subcutaneous hematoma $(n=3)$, wound infections $(n=5)$, wound dehiscence $(n=4)$, and post-operative neuroma related to graft harvest $(n=1)$. Significant post-operative pain was reported by 34 patients. These patients experienced symptomatic hardware $(n=19)$ or persistent knee pain $(n=15)$.

A pooled comparison of overall complication rates for knees that underwent tunnel fixation and those that underwent suture fixation can be seen in Figure 2. Studies that failed to clearly define their fixation technique as either suture or tunnel were excluded from this analysis. Because only one study ${ }^{25}$ directly compared the 2 fixation methods and because of the tremendous variation in study populations, procedures, and duration of follow-up, no clear statistical comparison can be made between the two fixation methods.

Similarly, no clear comparisons can be made regarding the occurrence of complications relative to the angle of knee flexion at the time of reconstruction (Figure 3). The reporting of knee angle varied across studies, with some studies reporting precise positions, others reporting a range of positions, and some studies failing to document knee flexion angle. As a result, knee flexion angle was dichotomized into 2 groups: those studies reporting positioning of the knee in less than $60^{\circ}$ of flexion, and those reporting knee flexion angles of $60^{\circ}$ or greater. Those studies that failed to describe knee flexion angle were excluded from the analysis of complications by knee flexion angle.

\section{Discussion}

Our group found 25 articles reporting MPFL reconstruction for patellar instability. Only 2 studies were classified as Level II evidence; none were Level I. This indicates a paucity of high-level evidence to evaluate the success and failure of MPFL reconstructions. Given that MPFL reconstructions have become a popular surgical procedure, this is somewhat concerning as this study found that the overall complication rate is not trivial. With an overall cumulative complication rate of $26.1 \%$, caution is indicated when this procedure is performed. Major complications included patellar fracture, post-operative instability, flexion loss, and pain. Many patients returned to the operative suite for manipulations to address decreased range of motion and removal of symptomatic hardware.

A large proportion of the complications were recurrent apprehension $(52 / 164=\sim 32 \%$ of all complications). It is unclear whether patients with recurrent instability had a failure due to graft loosening, rupture, or failure to recognize additional risk factors for recurrent patella 
instability. It is possible that the rate of recurrent apprehension and subluxation may be due to other unrecognized and uncorrected risk factors for patellofemoral instability such as an increased tibial tubercle-trochlear groove measurement, patella alta, or higher grade trochlea dysplasia. This underlines the importance of recognizing additional risk factors in patients with recurrent patella subluxations and dislocations. We suggest that authors should be held to report on the perceived reasons for failure as the various reported techniques each have numerous and unique reasons for potential failure of the graft.

The MPFL graft fixation methods vary within the literature but can generically be categorized as suture or tunnel techniques. Figure 2 provides descriptive data regarding the complication rate observed among both tunnel and suture techniques. A trend of more overall complications was observed utilizing the tunnel techniques (29.8\%) compared to suture techniques $(21.6 \%)$. However, the suture techniques demonstrated a higher rate of recurrent dislocation/subluxation (4.8\%) and apprehension/hypermobility (24.0\%) than the tunnel technique (3.3\% and $8.6 \%$, respectively). While this information raises questions regarding complication risk associated with each procedure, clear comparisons between the procedures cannot be made because of the relatively small sample sizes available, the variety of concomitant procedures performed with MPFL reconstruction, a lack of uniform reporting of complications, and variations in length of follow-up.

Arguably the most severe complication reported was a post-operative patella fracture. Four patella fractures were reported, all in patients who underwent MPFL reconstructions using single or double transverse bone tunnels $(n=429)$. The range of bone tunnels created in these 4 patients ranges from $3.2-\mathrm{mm}$ to $4.5-\mathrm{mm}$. One patient sustained a transverse patella fracture after a fall. His/Her fixation called for a transverse $3.2 \mathrm{~mm}$ patellar tunnel with a polyester graft that was fixed to the lateral patella using a knot. Two patients had a $4.5-\mathrm{mm}$ patellar tunnel that exited the anterior patella. The authors used a semitendinosus autograft with the free end passed through the tunnel, folded over, and sutured into place. A fourth patient developed a post-operative patella fracture when rising from a chair. The surgeons looped a gracilis graft through two 4.5 -mm transverse patella tunnels separated by 10 to 15 $\mathrm{mm}$. No patella fractures were reported in studies using a docking technique, anchors or a soft tissue attachment on the patella $(n=125)$. Even though the incidence of patella fracture is certainly not high enough to draw statistically relevant conclusions, it behooves the surgeon to consider using a technique that does not carry the inherent risk of fracture. In this context, a docking anchor based or suture fixation could be safer to use.

Another frequently reported complication after MPFL reconstruction is the loss of knee flexion. This was reported in 22 patients (13.4\% of all complications), out of which half required post-operative manipulation under anesthesia. The MPFL is a non-isometric ligament which acts primarily as a restraint. The concept of "tensioning" of the MPFL at any reported flexion angle therefore may be a conceptual problem. In its native state the MPFL is not under tension; it only comes under tension when a laterally displacing force acts on the patella. Therefore, "tensioning" the MPFL graft may in fact restrict range of motion. In addition, it may be important to clearly determine the femoral point of fixation intraoperatively according to easily identifiable landmarks. In many patients, it is very hard to clearly palpate the adductor tubercle. A reliable method described by Schottle et al. ${ }^{34}$ used radiographic landmarks that can easily be found under fluoroscopy. Schottle et al.'s point is $1-\mathrm{mm}$ anterior to the posterior cortex extension line, $2.5-\mathrm{mm}$ distal to the posterior origin of the medial femoral condyle, and proximal to the posterior point of the Blumensaat line on the lateral radiograph. ${ }^{34}$ However, a recent study showed potential anisometry of the MPFL graft related to the degree of patella alta. Tateishi et al. ${ }^{34}$ had 10 patients with average patellar height ratio of $1.4+/-0.2$ whose graft length increased 3 to 5.5 -mm with knee range of motion. The femoral fixation was near but not exactly at Schottle et al.'s 
specifications. ${ }^{39}$ For this group, the femoral tunnel averaged $-1.2+/-5.6-\mathrm{mm}$ relative to the posterior cortex, $4.9+/-2.1-\mathrm{mm}$ distal to the posterior origin of the medial femoral condyle, and $3.1+/-1.5-\mathrm{mm}$ proximal to the Blumensaat line.

Fixation at various flexion angles has been recommended. The angle of knee flexion at the time of graft placement may play a role in postoperative patellar stability. Figure 3 provides descriptive data for the influence of knee flexion angle on complication rate. A trend of those undergoing fixation at less than $60^{\circ}$ knee flexion $(32.6 \%, \mathrm{n}=230 \mathrm{knees})$ and experiencing a higher overall complication rate than those undergoing fixation at $60^{\circ}$ or greater knee flexion $(23.8 \%, \mathrm{n}=319$ knees) was observed. Similarly, a lower rate of recurrent subluxation/dislocation (1.6\% vs. $6.1 \%)$ and continued apprehension/hypermobility $(9.1 \%$ vs. $9.6 \%$ ) was observed among those undergoing fixation at $60^{\circ}$ or greater compared to those undergoing fixation at less than $60^{\circ}$. However, caution is urged in interpreting these values as they are only descriptive in nature and previously discussed limitations prevent direct statistical comparison. At full extension the medial retinaculum and MFPL is most taught and resists patellar subluxation. ${ }^{2}$ The patella subluxes most easily at $20^{\circ}$ of flexion. ${ }^{35}$ As knee flexion increases, the medial retinaculum slackens and the femoral trochlea limits medial and lateral displacement of the patella. Previous work also reports that maximal graft length occurs at $60^{\circ}$ flexion. ${ }^{37}$ Some authors advocate graft fixation at increased knee flexion angles to prevent over tightening of the graft ${ }^{1}$ while others call for fixation at decreased knee flexion because the maximal effect of the MPFL occurs from 0 to 20 degrees. ${ }^{32}$ This area requires further comparative investigations before conclusions can be drawn.

This study revealed that a significant number of patients had to return to the operating room for additional surgical procedures. The highest number of secondary surgeries was reported for manipulations under anesthesia (1.4\%), for loss of knee motion, and for removal of symptomatic hardware (1.1\%). We addressed the concerns regarding the loss of range of motion in the above paragraph. One should also be aware of the fact that any hardware at the edge of the patella or the medial side of the knee may become prominent once the surgical swelling has resolved. Patients may tolerate hardware in these areas less well than in other areas of the knee (e.g., proximal tibia after anterior cruciate ligament reconstructions) thus requesting hardware removal. A total of 19 patients across 5 studies complained of painful hardware. ${ }^{6,8,16,26,38}$ Of these 5 studies, 133 patients underwent MPFL reconstruction with metallic implants. In one study some patients in the cohort did not have implanted hardware and were excluded from the previous total. ${ }^{38}$ Twelve patients experienced symptomatic staples, 3 had painful lag screws, and 3 had symptomatic interference screws. All of these implants were located on the femoral side. One patient had symptomatic hardware related to TTT.

The ability to draw conclusions from this review is greatly limited because of a lack of uniform reporting of methodology across the included studies. Significant variability was observed in the complication rate reported in individual studies with complications rates ranging from $0 \%$ to $85.2 \% .{ }^{26}$ Similarly, the large standard deviations for the mean rates of recurrent subluxation/dislocation and continued hypermobility/apprehension suggest that complication rates were highly variable between the included studies and not normally distributed. This variability is likely not the result of tremendous variations in the overall clinical outcome between studies, but rather is a function of the variation in study methodology for the reporting of complications and length of follow-up.

In conclusion, reconstruction of the MPFL overall is a popular procedure that can yield successful outcomes in many patients. However, despite its popularity, it is a procedure that can be associated with significant intra and post-operative complications that should be 
considered prior to choosing a technique. Also, there is a paucity of high-level studies evaluating MPFL reconstruction techniques, thus limiting our ability to judge the true outcome of this procedure with regards to complication rate. Further high-level studies with uniform reporting of methodology and clinical outcomes including complications are needed to detect the overall outcome, risks and benefits of this procedure. In particular, it will be necessary to better define clinical failure versus success based upon clinical, radiographic and patient reported outcomes parameters.

\section{References}

1. Ahmad CS, Brown GD, Stein BS. The docking technique for medial patellofemoral ligament reconstruction: surgical technique and clinical outcome. Am J Sports Med. 2009; 37(10):20212027. [PubMed: 19546481]

2. Amis AA, Firer P, Mountney J, Senavongse W, Thomas NP. Anatomy and biomechanics of the medial patellofemoral ligament. Knee. 2003; 10(3):215-220. [PubMed: 12893142]

3. Baldwin JL. The anatomy of the medial patellofemoral ligament. Am J Sports Med. 2009; 37(12): 2355-2361. [PubMed: 19729366]

4. Beasley LS, Vidal AF. Traumatic patellar dislocation in children and adolescents: treatment update and literature review. Curr Opin Pediatr. 2004; 16(1):29-36. [PubMed: 14758111]

5. Carson WG Jr. James SL, Larson RL, Singer KM, Winternitz WW. Patellofemoral disorders: physical and radiographic evaluation. Part II: Radiographic examination. Clin Orthop Relat Res. 1984; (185):178-186. [PubMed: 6705376]

6. Christiansen SE, Jacobsen BW, Lund B, Lind M. Reconstruction of the medial patellofemoral ligament with gracilis tendon autograft in transverse patellar drill holes. Arthroscopy. 2008; 24(1): 82-87. [PubMed: 18182207]

7. Conlan T, Garth WP Jr. Lemons JE. Evaluation of the medial soft-tissue restraints of the extensor mechanism of the knee. J Bone Joint Surg Am. 1993; 75(5):682-693. [PubMed: 8501083]

8. Cossey AJ, Paterson R. A new technique for reconstructing the medial patellofemoral ligament. Knee. 2005; 12(2):93-98. [PubMed: 15749442]

9. Deie M, Ochi M, Sumen Y, Adachi N, Kobayashi K, Yasumoto M. A long-term follow-up study after medial patellofemoral ligament reconstruction using the transferred semitendinosus tendon for patellar dislocation. Knee Surg Sports Traumatol Arthrosc. 2005; 13(7):522-528. [PubMed: 15968532]

10. Deie M, Ochi M, Sumen Y, Yasumoto M, Kobayashi K, Kimura H. Reconstruction of the medial patellofemoral ligament for the treatment of habitual or recurrent dislocation of the patella in children. J Bone Joint Surg Br. 2003; 85(6):887-890. [PubMed: 12931813]

11. Desio SM, Burks RT, Bachus KN. Soft tissue restraints to lateral patellar translation in the human knee. Am J Sports Med. 1998; 26(1):59-65. [PubMed: 9474403]

12. Dopirak R, Adamany D, Bickel B, Steensen R. Reconstruction of the medial patellofemoral ligament using a quadriceps tendon graft: a case series. Orthopedics. 2008; 31(3):217. [PubMed: 19292257]

13. Drez D Jr. Edwards TB, Williams CS. Results of medial patellofemoral ligament reconstruction in the treatment of patellar dislocation. Arthroscopy. 2001; 17(3):298-306. [PubMed: 11239352]

14. Ellera Gomes JL. Medial patellofemoral ligament reconstruction for recurrent dislocation of the patella: a preliminary report. Arthroscopy. 1992; 8(3):335-340. [PubMed: 1418205]

15. Ellera Gomes JL, Stigler Marczyk LR, Cesar de Cesar P, Jungblut CF. Medial patellofemoral ligament reconstruction with semitendinosus autograft for chronic patellar instability: a follow-up study. Arthroscopy. 2004; 20(2):147-151. [PubMed: 14760346]

16. Fernandez E, Sala D, Castejon M. Reconstruction of the medial patellofemoral ligament for patellar instability using a semitendinosus autograft. Acta Orthop Belg. 2005; 71(3):303-308. [PubMed: 16035703]

17. Fithian DC, Paxton EW, Stone ML, et al. Epidemiology and natural history of acute patellar dislocation. Am J Sports Med. 2004; 32(5):1114-1121. [PubMed: 15262631] 
18. Gomes JE. Comparison between a static and a dynamic technique for medial patellofemoral ligament reconstruction. Arthroscopy. 2008; 24(4):430-435. [PubMed: 18375275]

19. Han H, Xia Y, Yun X, Wu M. Anatomical transverse patella double tunnel reconstruction of medial patellofemoral ligament with a hamstring tendon autograft for recurrent patellar dislocation. Arch Orthop Trauma Surg. 2011; 131(3):343-351. [PubMed: 20714901]

20. Hautamaa PV, Fithian DC, Kaufman KR, Daniel DM, Pohlmeyer AM. Medial soft tissue restraints in lateral patellar instability and repair. Clin Orthop Relat Res. 1998; (349):174-182. [PubMed: 9584380]

21. Hawkins RJ, Bell RH, Anisette G. Acute patellar dislocations. The natural history. Am J Sports Med. 1986; 14(2):117-120. [PubMed: 3717480]

22. Heegaard J, Leyvraz PF, Van Kampen A, Rakotomanana L, Rubin PJ, Blankevoort L. Influence of soft structures on patellar three-dimensional tracking. Clin Orthop Relat Res. 1994; (299):235243. [PubMed: 8119024]

23. Matthews JJ, Schranz P. Reconstruction of the medial patellofemoral ligament using a longitudinal patellar tunnel technique. Int Orthop. 34(8):1321-1325. [PubMed: 19997732]

24. Mikashima Y, Kimura M, Kobayashi Y, Asagumo H, Tomatsu T. Medial patellofemoral ligament reconstruction for recurrent patellar instability. Acta Orthop Belg. 2004; 70(6):545-550. [PubMed: 15669454]

25. Mikashima Y, Kimura M, Kobayashi Y, Miyawaki M, Tomatsu T. Clinical results of isolated reconstruction of the medial patellofemoral ligament for recurrent dislocation and subluxation of the patella. Acta Orthop Belg. 2006; 72(1):65-71. [PubMed: 16570897]

26. Nomura E, Horiuchi Y, Kihara M. A mid-term follow-up of medial patellofemoral ligament reconstruction using an artificial ligament for recurrent patellar dislocation. Knee. 2000; 7(4):211215. [PubMed: 11104912]

27. Nomura E, Inoue M. Hybrid medial patellofemoral ligament reconstruction using the semitendinous tendon for recurrent patellar dislocation: minimum 3 years' follow-up. Arthroscopy. 2006; 22(7):787-793. [PubMed: 16843816]

28. Nomura E, Inoue M, Kobayashi S. Long-term follow-up and knee osteoarthritis change after medial patellofemoral ligament reconstruction for recurrent patellar dislocation. Am J Sports Med. 2007; 35(11):1851-1858. [PubMed: 17724092]

29. Nomura E, Inoue M, Osada N. Anatomical analysis of the medial patellofemoral ligament of the knee, especially the femoral attachment. Knee Surg Sports Traumatol Arthrosc. 2005; 13(7):510515. [PubMed: 15895206]

30. Obremskey W, Pappas N, Attallah-Wasif E, Tornetta P, Bhandari M. Levels of Evidence in Orthopaedic Journals. J Bone Joint Sur Am. 2005; 87(12):2632-2638.

31. Philippot R, Chouteau J, Wegrzyn J, Testa R, Fessy MH, Moyen B. Medial patellofemoral ligament anatomy: implications for its surgical reconstruction. Knee Surg Sports Traumatol Arthrosc. 2009; 17(5):475-479. [PubMed: 19225760]

32. Ronga M, Oliva F, Longo UG, Testa V, Capasso G, Maffulli N. Isolated medial patellofemoral ligament reconstruction for recurrent patellar dislocation. Am J Sports Med. 2009; 37(9):17351742. [PubMed: 19470945]

33. Schottle PB, Fucentese SF, Romero J. Clinical and radiological outcome of medial patellofemoral ligament reconstruction with a semitendinosus autograft for patella instability. Knee Surg Sports Traumatol Arthrosc. 2005; 13(7):516-521. [PubMed: 15959766]

34. Schottle PB, Romero J, Schmeling A, Weiler A. Technical note: anatomical reconstruction of the medial patellofemoral ligament using a free gracilis autograft. Arch Orthop Trauma Surg. 2008; 128(5):479-484. [PubMed: 17323067]

35. Senavongse W, Amis AA. The effects of articular, retinacular, or muscular deficiencies on patellofemoral joint stability. J Bone Joint Surg Br. 2005; 87(4):577-582. [PubMed: 15795215]

36. Sillanpaa P, Mattila VM, Visuri T, Maenpaa H, Pihlajamaki H. Ligament reconstruction versus distal realignment for patellar dislocation. Clin Orthop Relat Res. 2008; 466(6):1475-1484. [PubMed: 18347890]

37. Smirk C, Morris H. The anatomy and reconstruction of the medial patellofemoral ligament. Knee. 2003; 10(3):221-227. [PubMed: 12893143] 
38. Steiner TM, Torga-Spak R, Teitge RA. Medial patellofemoral ligament reconstruction in patients with lateral patellar instability and trochlear dysplasia. Am J Sports Med. 2006; 34(8):1254-1261. [PubMed: 16567459]

39. Tateishi T, Tsuchiya M, Motosugi N, et al. Graft length change and radiographic assessment of femoral drill hole position for medial patellofemoral ligament reconstruction. Knee Surg Sports Traumatol Arthrosc. 2011; 19(3):400-407. [PubMed: 20811734]

40. Thaunat M, Erasmus PJ. The favourable anisometry: an original concept for medial patellofemoral ligament reconstruction. Knee. 2007; 14(6):424-428. [PubMed: 17933540]

41. Toritsuka Y, Amano H, Mae T, et al. Dual tunnel medial patellofemoral ligament reconstruction for patients with patellar dislocation using a semitendinosus tendon autograft. Knee. 2011; 18(4): 214-219. [PubMed: 20684880]

42. Tuxoe JI, Teir M, Winge S, Nielsen PL. The medial patellofemoral ligament: a dissection study. Knee Surg Sports Traumatol Arthrosc. 2002; 10(3):138-140. [PubMed: 12012030]

43. Watanabe T, Muneta T, Ikeda H, Tateishi T, Sekiya I. Visual analog scale assessment after medial patellofemoral ligament reconstruction: with or without tibial tubercle transfer. J Orthop Sci. 2008; 13(1):32-38. [PubMed: 18274853] 


\begin{tabular}{|c|c|}
\hline 146 total articles & 43 described other surgeries \\
\hline 103 MPFL articles & -27 anatomy studies \\
\hline 76 MPFL articles & -7 editorial/letters \\
\hline 69 MPFL surgery articles & -3 MPFL repair papers \\
\hline 66 MPFL reconstruction articles & 9 review papers \\
\hline 57 MPFL reconstruction articles & - 15 technical notes \\
\hline $42 \mathrm{MPFL}$ reconstruction articles & -6 case reports \\
\hline 36 MPFL reconstruction articles with outcomes & -10 non-English papers \\
\hline 26 English MPFL reconstruction articles with outcomes & -1 did not report complications \\
\hline
\end{tabular}

Figure 1.

Article elimination flowchart 


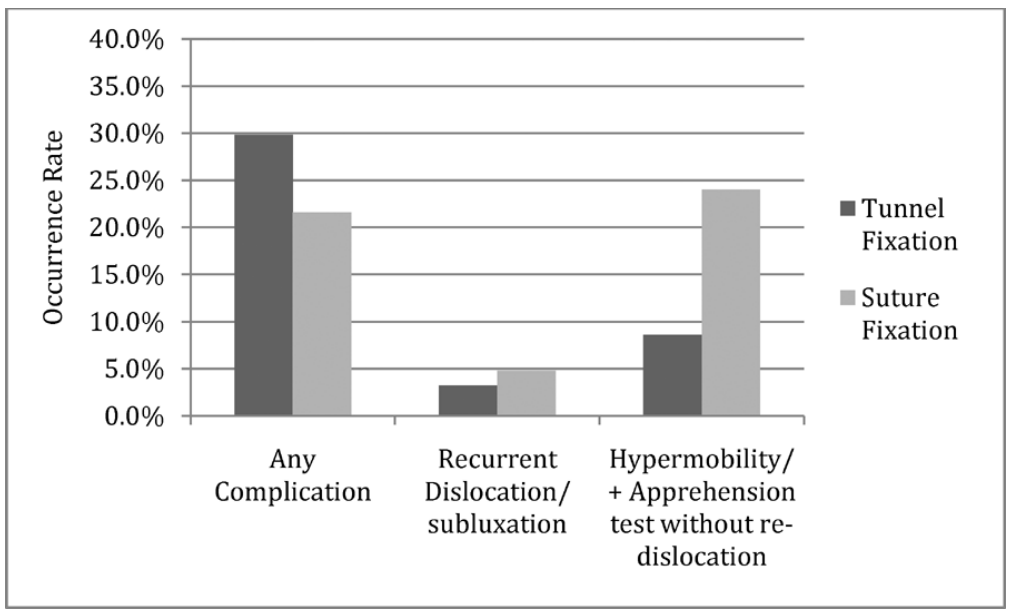

Figure 2.

Occurrence of Complication by Fixation Technique 


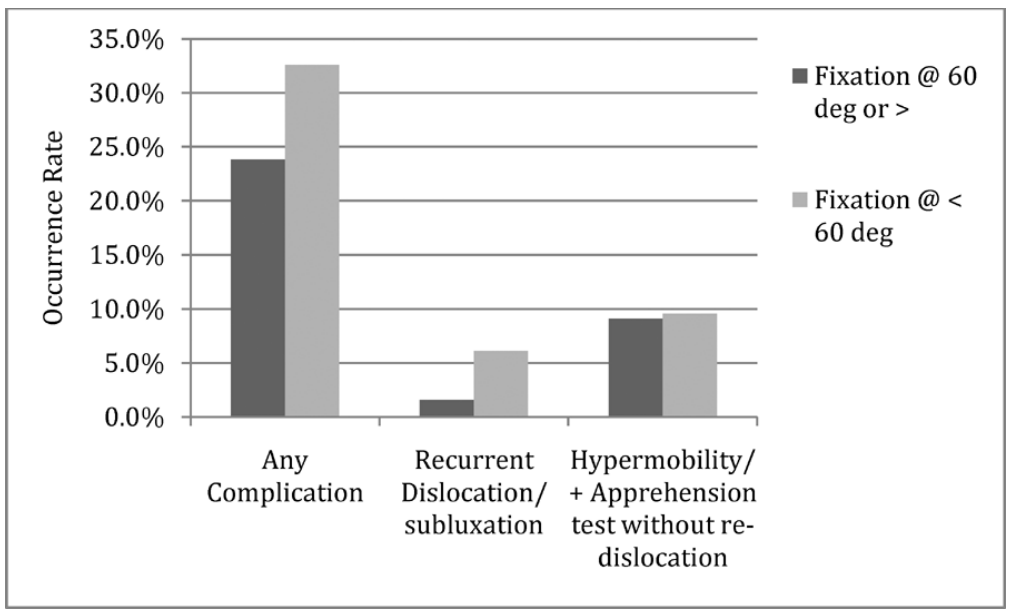

Figure 3.

Occurrence of Complications by Fixation Angle 